\title{
Vapour-liquid equilibria of the hard core Yukawa fluid
}

\author{
By B. SMIT \\ Koninklijke/Shell-Laboratorium, Amsterdam (Shell Research B.V.) Badhuisweg 3, \\ $1031 \mathrm{CM}$ Amsterdam, The Netherlands \\ D. FRENKEL \\ FOM-Institute for Atomic and Molecular Physics, Kruislaan 407, \\ 1098 SJ Amsterdam, The Netherlands \\ (Received 3 December 1990; accepted 20 March 1991) \\ Techniques which extend the range of applicability of the Gibbs ensemble \\ technique for particles which interact with a hard core potential are described. \\ The power of the new technique is demonstrated in a numerical study of the \\ vapour-liquid coexistence curve of the hard core Yukawa fluid.
}

\section{Introduction}

Some systems of practical importance, in particular certain colloids, are modelled with a hard core potential and an attractive tail [1]. Such a model is able to reproduce an important characteristic of real colloids, namely the occurrences of a 'liquidvapour' phase transition. However, the numerical simulation of this liquid-vapour coexistence has, thus far, proved difficult because the Gibbs ensemble technique [2] becomes quite inefficient for models that combine hard core repulsion with strong short ranged attraction. A case in point is the hard core Yukawa model. A modified simulation scheme is proposed which takes advantage of the specific properties of the hard core potential. This technique significantly increases the efficiency of the Gibbs ensemble technique for the class of models mentioned above. As an illustration, we report the numerical study of the vapour-liquid coexistence curve of the hard core Yukawa fluid.

Consider a fluid with a pairwise additive potential

$$
u(r)= \begin{cases}\infty, & r<\sigma, \\ w(r), & r>\sigma .\end{cases}
$$

in which $\sigma$ is the diameter of the hard core. For such a fluid Lebowitz and Percus [3] have developed the mean spherical approximation (MSA). Together with the exact condition

$$
h(r)=g(r)-1=-1, \quad r<\sigma
$$

the MSA consists in assuming that the direct correlation function [4] can be approximated by

$$
c(r)=-\beta w(r), \quad r>\sigma
$$

where $\beta=1 / k_{\mathrm{B}} T$. 
The importance of this theory is that for various model fluids the MSA combined with the Ornstein-Zernike equation [4] can be solved analytically. Examples of such fluids are the dipolar hard sphere fluid [5] and the hard core Yukawa fluid [6].

The tail of the hard core Yukawa fluid is given by

$$
w(r)=-\varepsilon \frac{\exp [-\lambda(r-\sigma)]}{r / \sigma}, \quad r>\sigma .
$$

Several authors [7-10] have studied this fluid on the basis of the MSA results. Expressions for the density profile of this fluid in a simple external field of an idealized wall have been obtained [11-13]. These applications make this fluid interesting as a starting point for various perturbation theories for other model fluids [14]. It is, therefore, important to have 'exact' numerical data on the phase behaviour of this fluid.

For the hard-core Yukawa fluid the Gibbs ensemble technique cannot be applied straightforwardly as pointed out by Rudisill and Cummings [2]. Since it is energetically favourable for two particles to be in contact, a decrease of the volume will most likely result in an overlap of a least one pair. The probability of a volume change to be accepted will, therefore, be extremely small and it would require a very large number of these volume steps in order to obtain a proper estimate of the coexistence properties. Since the potential does not scale with the volume, a volume step (if implemented straightforwardly) is a relatively 'expensive' operation. It would, therefore, be questionable whether the Gibbs ensemble technique is 'cheaper' than conventional methods for obtaining phase equilibrium data for this type of fluid.

In this paper we demonstrate that for the hard core fluids one can utilize some of the techniques developed by Wood [15] for constant pressure simulations of hard discs, to reduce the computer costs for the volume step significantly.

\section{Gibbs ensemble simulations}

Details on the Gibbs ensemble simulation technique can be found in [16, 17]. A formal proof of the equivalence of the Gibbs ensemble and the canonical ensemble is given in [18]. We have used the algorithm described in $[19,20]$ which deviates slightly from the original algorithm of Panagiotopoulos [16]. Below we list the extensions for a hard-core potential.

In case of a hard core fluid, a configuration can be rejected if an overlap between a pair of particles has been detected. Since it is usually much cheaper to detect such an overlap than to calculate the energy of a configuration, one can take advantage of this in several steps of the Gibbs ensemble.

\subsection{Particle displacement}

During the simulations for each particle a neighbour list was made containing all particles within a radius equal to the maximum displacement. Before the energy of the new configuration was calculated, this neighbour list was used to test for a possible overlap. This makes the computer time required for a rejected move much less than the time required for an accepted move. The maximum displacement was, therefore, set to give an acceptance ratio of approximately $25 \%$.

\subsection{Volume change}

During the simulations we kept track of the minimum distance between a pair of particles in each box. When, after a volume change, one of these minima becomes 
smaller than the hard core radius the configuration can be rejected immediately. We performed one attempt to change the volume after each Monte Carlo step.

Since the hard core Yukawa potential is monotonically increasing for distances greater than the hard core diameter, an increase of the volume will always result in an increase of the total energy. This makes it unavoidable to use relatively small volume changes.

Because of the low probability of acceptance, even with a relatively small volume change, it is important to perform a very large number of volume changes during each cycle of the Gibbs ensemble simulation. Although the overlap criterion will reject, for a given maximum volume change, typically 90 per cent of these configurations, the calculation of the energy of the remaining 10 per cent would still require a very large amount of CPU time.

In order to reduce the required CPU time further, a method was developed to calculate the energy of a new configuration more efficiently.

The total energy $(U)$ of the old configuration is given by

$$
U=\frac{1}{2} \sum_{i, j} u\left(r_{i j}\right)
$$

where $r_{i j}$ is the distance between the particles $i$ and $j$ and $u(r)$ is the intermolecular potential as defined by (1) and (4). Assume that a volume change is achieved by rescaling of the coordinates

$$
r^{\prime}=(1+\Delta) r
$$

where the prime indicates the new configuration. If we furthermore assume that there is no overlap between two particles, the total energy of this new configuration is given byt

$$
U^{\prime}=\frac{1}{2} \sum_{i, j} \frac{\exp \left[-\lambda\left(r_{i j}^{\prime}-1\right)\right]}{r_{i j}^{\prime}}=\frac{1}{2} \sum_{i, j} \frac{\exp \left[-\lambda\left((1+\Delta) r_{i j}-1\right)\right]}{(1+\Delta) r_{i j}} .
$$

Since the volume change is small, we can make a Taylor expansion of the exponent [21]

$$
U^{\prime}(\Delta)=\frac{U}{1+\Delta}+\sum_{n=1}^{\infty} c_{n} \frac{\Delta^{n}}{1+\Delta}
$$

with

$$
c_{\mathrm{n}}=\frac{1}{2} \frac{-1^{n}}{n !} \lambda^{n} \sum_{i, j} \frac{\exp \left[-\lambda\left(r_{i j}-1\right)\right]}{r_{i j}} r_{i j}^{n} .
$$

The coefficients $c_{\mathrm{n}}$ depend only on the coordinates of the old configuration. Tests show that four terms in this expansion estimate the energy of the new configuration with a relative error of approximately $10^{-6}$. Updating the coefficients $c_{\mathrm{n}}$ during a simulation does not require much CPU time and therefore this method allows us to calculate the energy of a new configuration very efficiently. However, once a new configuration has been accepted the new coefficients $c_{n}$ need to be calculated, which is still a relatively expensive operation.

Before each simulation was started a short test run was performed to estimate the maximum volume change which gave the maximum mean squared volume change per unit CPU.

$\dagger$ In the rest of this paper the energy is in units of $\varepsilon$ and the length in units of $\sigma$. 


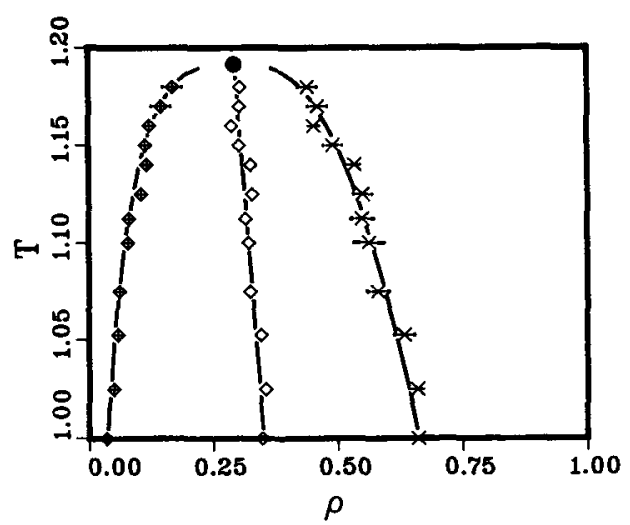

Phase diagram of the hard core Yukawa fluid $(\lambda=1 \cdot 8) \times, \diamond$, and $\oplus$ are the Gibbs ensemble results of this work. $\bullet$ is the estimate of the critical point on the basis of the simulation results. The full lines are the fits to the scaling law and the rectilinear law. The temperature and density are in the usual reduced units.

\subsection{Particle exchange}

Except for some extra book keeping for updating the neighbour lists, the minimum distances between pairs in each box and the coefficients $c_{n}$, the particle exchange step was not modified.

\section{Results of the simulations}

We have calculated the phase diagram for $\lambda=1.8$. The contributions of the tail of the potential was estimated analytically assuming $g(r)=1$ for distances greater

Results for the hard-core Yukawa fluid $\lambda=1 \cdot 8 . N$ is the total number of particles, $T$ the temperature, $N_{\mathrm{cy}}$ is the number of Monte Carlo cycles, $\rho$ the density, $P$ the pressure, $E$ the energy, and $\mu$ the chemical potential, all in the usual reduced units. The number of attempts per cycle to insert a particle were: $N_{\text {try }}=40$. The small subscripts give the accuracy of the last digit(s), so 0.731 , means $0.731 \pm 0.007$.

\begin{tabular}{|c|c|c|c|c|c|c|c|c|c|c|}
\hline \multirow[b]{2}{*}{$N$} & \multirow[b]{2}{*}{$T$} & \multirow[b]{2}{*}{$\frac{N_{\mathrm{cy}}}{10^{3}}$} & \multicolumn{4}{|c|}{ Gas phase } & \multicolumn{4}{|c|}{ Liquid phase } \\
\hline & & & $\rho_{\mathrm{g}}$ & $P_{\mathrm{g}}$ & $-E_{\mathrm{g}}$ & $-\mu_{\mathrm{g}}$ & $\rho_{1}$ & $P_{1}$ & $-E_{1}$ & $-\mu_{\mathrm{i}}$ \\
\hline 108 & 1.000 & 5 & $0.036_{6}$ & $0.030_{4}$ & $0 \cdot 3_{1}$ & $3 \cdot 60_{10}$ & $0.66_{1}$ & $0 \cdot 0_{1}$ & $4 \cdot 5_{I}$ & $3 \cdot 56_{22}$ \\
\hline 108 & 1.025 & 10 & $0.050_{7}$ & $0.040_{4}$ & $0 \cdot 4_{1}$ & $3 \cdot 47_{8}$ & $0.66_{1}$ & $0 \cdot 0_{1}$ & $4 \cdot 5_{2}$ & $3 \cdot 39_{23}$ \\
\hline 108 & $1 \cdot 053$ & 10 & $0.059_{9}$ & $0 \cdot 047_{7}$ & $0 \cdot 4_{3}$ & $3 \cdot 44_{9}$ & $0.63_{2}$ & $0.03_{6}$ & $4 \cdot 3_{3}$ & $3.41_{16}$ \\
\hline 108 & 1.075 & 10 & $0.06_{s}$ & $0.051_{3}$ & $0 \cdot 46_{6}$ & $3 \cdot 48_{6}$ & $0.58_{2}$ & $0.0_{7}$ & $3 \cdot 9_{2}$ & $3.48_{9}$ \\
\hline 108 & $1 \cdot 100$ & 10 & $0.08_{1}$ & $0.067_{8}$ & $0 \cdot 6_{1}$ & $3 \cdot 37_{5}$ & $0.56_{3}$ & $0.03_{9}$ & $4 \cdot 0_{2}$ & $3 \cdot 34_{8}$ \\
\hline 108 & $1 \cdot 112$ & 10 & $0.08_{1}$ & $0.059_{s}$ & $0 \cdot 6_{2}$ & $3 \cdot 42_{6}$ & $0.55_{2}$ & $0.06_{7}$ & $3 \cdot 7_{2}$ & $3 \cdot 38_{7}$ \\
\hline 108 & $1 \cdot 125$ & 7 & $0 \cdot 106_{13}$ & $0.076_{8}$ & $0 \cdot 8_{2}$ & $3 \cdot 31_{4}$ & $0.550_{19}$ & $0.06_{8}$ & $3 \cdot 7_{2}$ & $3 \cdot 29_{5}$ \\
\hline 108 & $1 \cdot 140$ & 7 & $0 \cdot 118_{11}$ & $0.080_{5}$ & $0.9_{2}$ & $3 \cdot 30_{3}$ & $0.534_{15}$ & $0.07_{s}$ & $3 \cdot 6_{2}$ & $3 \cdot 33_{4}$ \\
\hline 108 & $1 \cdot 150$ & 7 & $0 \cdot 115_{9}$ & $0.084_{9}$ & $0 \cdot 9_{1}$ & $3 \cdot 32_{3}$ & $0.491_{19}$ & $0.07_{5}$ & $3 \cdot 3_{2}$ & $3 \cdot 33_{3}$ \\
\hline 108 & $1 \cdot 160$ & 5 & $0 \cdot 124_{18}$ & $0.085_{11}$ & $0 \cdot 9_{3}$ & $3 \cdot 32_{4}$ & $0.454_{14}$ & $0.08_{4}$ & $3 \cdot I_{2}$ & $3 \cdot 34_{s}$ \\
\hline 108 & $1 \cdot 170$ & 7 & $0 \cdot 146_{20}$ & $0.080_{5}$ & - & $3 \cdot 26_{3}$ & $0.464_{20}$ & $0.07_{s}$ & - & $3 \cdot 27_{2}$ \\
\hline 108 & $1 \cdot 180$ & 7 & $0 \cdot 17_{2}$ & $0.08_{1}$ & - & $3 \cdot 28_{3}$ & $0.44_{2}$ & $0 \cdot 09_{2}$ & - & $3 \cdot 28_{3}$ \\
\hline
\end{tabular}

boxes changed identity during the simulation. 
than half the box size. The pressure of the fluid in box $k$ was calculated using

$$
\left\langle P^{(k)}\right\rangle=T\left\langle\rho^{(k)}\right\rangle-\frac{1}{3 V}\left\langle\sum_{i<j}{ }^{(k)} r_{i j} \frac{\mathrm{d} w\left(r_{i j}\right)}{\mathrm{d} r}\right\rangle+\frac{2}{3} \pi T\left\langle\left(\rho^{(k)}\right)^{2}\right\rangle g^{(k)}\left(1^{+}\right)
$$

in which the summation in the second term on the right-hand side runs over all particles in box $k \cdot g^{(k)}\left(1^{+}\right)$denotes the radial distribution function at contact, which has been obtained by extrapolation. The formulae for the chemical potential that have been used are given in [22].

The results of the simulations are presented in the figure and in the table. The critical temperature and density have been estimated by fitting the results to the law of rectilinear diameters and to a scaling law for the densities [23, 24] (with the three-dimensional Ising critical exponent, $\beta=0.32$ ). The estimated critical temperature and density are $T_{\mathrm{c}}=1.192 \pm 0.008$ and $\rho_{\mathrm{c}}=0.294 \pm 0.006$.

Comparison of these results with the prediction of the mean spherical approximation, which give for the critical temperature and density $T_{\mathrm{c}}=1.05$ and $\rho_{\mathrm{c}}=0.32$ [9], shows that the MSA theory underestimates the critical temperature significantly.

\section{Summary}

In this paper we have presented an extension of the Gibbs ensemble technique for particles which interact with a hard core potential more efficiently. We have used these methods to calculate the vapour-liquid curve of the hard core Yukawa fluid.

The work of the FOM-Institute is part of the research program of FOM and is supported by 'Nederlandse Organisatie voor Wetenschappelijk Onderzoek' (NWO).

\section{References}

[1] Kranendonk, W., 1990, PhD thesis, Rijksuniversiteit Utrecht, The Netherlands.

[2] Rudisill, E. N. and Cummings, P. T., 1989, Molec. Phys., 68, 629.

[3] Lebowitz, J. L. and Percus, J. K., 1966, Phys. Rev., 144, 251.

[4] Hansen, J. P. and MCDonald, I. R., 1986, Theory of Simple Liquids. 2nd edn (Academic).

[5] Wertheim, M. S., 1971, J. chem. Phys., 55, 4291.

[6] Waisman, E., 1973, Molec. Phys., 25, 45.

[7] Henderson, D. and Blum, L., 1976, Molec. Phys., 32, 1627.

[8] Høye, J. S. and Stell, G., 1976, Molec. Phys., 32, 209.

[9] Cumming, P. T. and Smith, E. R., 1979, Chem. Phys., 42, 241.

[10] Cumming, P. T. and Smith, E. R., 1979, Molec. Phys., 38, 997.

[11] Waisman, E., Henderson, D. and Lebowitz, J. L., 1976, Molec. Phys., 32, 1373.

[12] Henderson, D., Abraham, F. F. and Barker, J. A., 1976, Molec. Phys., 31, 1291.

[13] Blum, L. and Stell, G., 1976, J. stat. Phys., 15, 439.

[14] Cumming, P. T., Wright, C. C. and Smith, E. R., 1979, Chem. Phys. Lett., 66, 278.

[15] Wood, W. W., 1970, J. chem. Phys., 52, 729-741.

[16] Panagiotopoulos, A. Z., 1987, Molec. Phys., 61, 813.

[17] Panagiotopoulos, A. Z., Quirke, N., Stapleton, M. and Tildesley, D. J., 1988, Molec. Phys., 63, 527.

[18] Smit, B., de Smedt, Ph. and Frenkel, D., 1989, Molec. Phys., 68, 931-950.

[19] Smit, B., 1990, PhD thesis, Rijksuniversiteit Utrecht, The Netherlands.

[20] Smit, B. and Frenkel, D., 1991, J. chem. Phys. 94, 5663.

[21] Adams, D. J. and McDonald, I. R., 1974, J. Phys. C, 7, 2761.

[22] Smit, B. and Frenkel, D., 1989, Molec. Phys., 68, 951-958.

[23] Rowlinson, J. S. and Swinton, F. L., 1982, Liquids and Liquid Mixtures. 3rd edn (Butterworth).

[24] Smit, B. and Williams, C. P., 1990, J. Phys. Condens. Matter, 2, 4281. 